\title{
Editorial Statistics 2020
}

Papers submitted

Of those papers were:

- rejected by editorial decision 103

- handed back to authors for revision (by editorial decision) 18

- submitted to double-blind review after initial revision 9

- rejected by editorial decision after initial revision 2

- rejected by unanimous reviewer decision

- withdrawn by the authors

- cancelled by editorial decision

- altered to Research Notes 4

- directly submitted to double-blind review 68

- altered to Research Notes

- rejected by unanimous reviewer decision 5

- rejected by editorial decision --

- withdrawn by the authors

- cancelled by editorial decision --

- Rejection rate: $\quad 57 \%$

- accepted for publication (after revision) 15

- published as Articles in 2020

- published as Research Notes in 2020

- scheduled to be published in 2021

Average feedback duration $\quad 53$

(i.e. time between submission of a paper and feedback): days

Feedback loops of more than 100 days $\quad 50$

(Editorial committee target line):

Reviews provided: 
We express our gratitude to the following reviewers for their support provided to our journal through their critical and fair reviews in 2020:

Ulun Akturan
Maria Aluchna
Özlem Araci
Maruthi Rohit Ayyagari
Norbert Bach
Gyula Bakacsi
Karoly Balaton
Yannick Bammens
Pieter-Jan Bezemer
Daniel Blab
Susanne Blazejewski
Suleika Bort
Carina Braun
Markus Braun
Wolfgang Buchholz
Jurgita Butkeviciene
Zoltan Buzady
Doina Catana
Barbara Čater
Tomaž Čater
Adam Chmielewski
Sven-Olof Collin
Cristian Dabija
Tobias Dauth
Eckhard Dittrich
Claudia Doblinger
Gregor Dorfleitner
Michael Dowling
Tiit Elenurm
Allen Engle
John Erpenbeck
Bengi Ertuna
Andreas Falke
Stuart Farquhar
Veronika Fetzer
Jan Hendrik Fisch
Barbara Frey
Hansjörg Gaus
Anja Geigenmüller




Mike Geppert
Eskil Goldeng
Victoria Golikova
Igor Gurkov
Nereida Hadziahmetovic
Axel Haller
Sebastian Händschke
Nina Katrin Hansen
Axel Haunschild
Stefan Hauptmann
Balázs Heidrich
Roland Helm
Niels Hermes
Stefan Hüsig
Dinh Tran Ngoc Huy
Steffen Jahn
Andreja Jaklic
Barbara Jankowska
Miroslaw Jarosinski
Antti Kauppinen
Eric Kearney
Elisabeth Kelan
Florian Kellner
Wafa Khlif
Kurmet Kivipõld
Mirjana Kljajic
Blaženka Kneževic
Romana Korez Vide
Mihai Korka
Marko Kosak
Arjan Kozica
Olaf Kranz
Bettina Kubicek
Irena Kustec
Anna Lašḱková
Shyqyri Llaci

Mike Geppert

Eskil Goldeng

Igor Gurkov

Axel Haller

Sebastian Händschke

Axel Haunschild

Stefan Hauptmann

Roland Helm

Niels Hermes

Steffen Jahn

Andreja Jaklic

Miroslaw Jarosinski

Antti Kauppinen

rney

Elisabeth Kelan

Wafa Khlif

Kurmet Kivipõld

Mirjana Kljajic

Romana Korez Vide

Mihai Korka

Arjan Kozica

tina Ku

Anna Lašákovã

Shyqyi Llaci
Stefan Schmid

Yvonne Schmid

Arnold Schuh

Klaus-Peter Schulz

Alma Sehic

Jurgita Sekliuckiene

Anna Sender

Galina Shirokova

Aram Simonyan

Anna Soulsby

Tobias Specker

Mohit Srivastava

Zivile Stankeviciute

Simona Sternad

Johannes Steyrer

Bernhard Swoboda

Erna Szabo

Katalin Takacs Haynes

Katharina Thill

Susanne Tietze

Piotr Trąpczyński

Ine Umans

Marco van Gelderen

Emil Velinov

Tiia Vissak

Krzysztof Wach

Gerhard Wagner

Peter Wald

Peter Walgenbach

Aleksandra Wasowska

Verena Watzek

Marius Wehner

Silvana Weiss

Michael Whitall

Ingo Winkler

Daniel Yar

Editorial Committee

Thomas Steger

Irma Rybnikova

Rainhart Lang 